\title{
Integrated Navigation Complex of UAV on Basis of Flight Controller
}

\author{
V.M. Sineglazov \\ Aviation Computer-Integrated Complexes Department \\ National Aviation University \\ Kyiv, Ukraine \\ svm@nau.edu.ua
}

\author{
V.P. Ischenko \\ Aviation Computer-Integrated Complexes Department \\ National Aviation University \\ Kyiv, Ukraine \\ IschenkoVitaly@gmail.com
}

\begin{abstract}
The problem of integrated navigation complex design of unmanned aerial vehicles is considered. It is determined the constitution of such navigation complexes. It is proposed its realization based on flight controller.
\end{abstract}

Keywords-unmanned aerial vehicle; integrated navigation complex; flight controller

\section{INTRODUCTION}

Currently, unmanned aerial vehicles (UAVs) have a special place among aircrafts. This is connected with their low cost, multifunctionality and ease of operation. Unmanned aerial vehicles are used for the solution of a variety of problems that previously were solved with help of manned aircrafts and helicopters.

Operation of UAVs in most cases is relatively cheap, and their low compared with MAs own cost and most importantly - the lack of people on board allows to send UAVs to the execution of tasks in which there is a significant risk of UAV loss. Initially, the UAV is controlled remotely from the earth (RC-model), but modern unmanned systems are increasingly equipped with an autopilot and an onboard computer, which allow them to solve the complicated tasks.

The necessity of autonomous operation can arise in cases where control of the UAV from the ground is difficult, for example, due to a large deletion of the terrain, the need of radio silence. Using autonomous UAVs allows also avoid the need of many hours manual piloting by man to a predetermined route or in cases where the final goal of flight is an aerial photography of the remote object.

In order to formulate the requirements for the navigation complex, you must determine the required functionality of the UAV.

\section{THE REQUIRED UAVS FUNCTIONALITY}

The main requirements that apply to UAVs are follows: high maneuverability, flight by day and at night under different weather conditions at the altitude $1000 \ldots 2500 \mathrm{~m}$ and speed $120 \ldots 240 \mathrm{~km} / \mathrm{h}$, the precision flight route with the determination own and target coordinates, the ability of the flight in the presence of electronic noise in a wide frequency range, the ability to control the payload (camera, thermal imager).
In addition, should take into account the requirements of the external pilot of the UAV:

- availability RTH (return to home) mode (flight mode in which the UAV will immediately stop flying and return to the point of take-off or the nearest point, which is considered the "home"), stabilization, cruise control, indications of current coordinates on the ground monitor, indications of the voltage across the two batteries, indications of flight speed, indications of altitude, the distance from the current position of the UAV to a point of take-off, the compass, the ability to fly in the winter;

- overboard temperature indications, the ability to connect antenna tracker (antenna station that amplifies the communication signals due with UAV) for longhaul flights, from $5 \mathrm{~km}$.

\section{JUSTIFICATION OF NECESSITY FOR CREATING INTEGRATED NAVIGATION COMPLEXES}

The general trend of mobile navigation systems market are such that under the influence of the increasingly stringent requirements the developers are moving along the path of greater integration between the inertial, satellite and other navigation systems. At the same time ICAO Committee on perspective navigation systems (FANS-Future Air Navigation System) recommends using the board Satellite Navigation Systems (SNS) under the necessary combination with an inertial navigation system as the central link in the navigation system.

However, in conditions of limited visibility of satellites as well as the loss of information from the satellite navigation system, for example, due to the difficult conditions of radio signals reception for acceptable navigation solution receiving the source data is not enough. Therefore, in this case, there is a risk of loss of UAV flight information support and as a consequence the loss of the UAV (rough strapdown inertial navigation system (SINS) is unable to provide the UAV the flight control and navigation information required accuracy even at small time intervals).

As an additional navigation UAVs systems can use the following navigation systems: Aeromagnetometric Navigation System (AMNS), Synthetic Aperture Radar Navigation System (SARNS), Terrain-Referenced Navigation System (TRNS), Landscape Navigation System (LNS). 
In this work in order to minimize costs and achieve high accuracy and reliable indicators emphasis on modular principle of Flight and Navigation Complex (FNC) design, when each of the above navigation systems is implemented as a separate module, which are adapted to optimally selected for solving the problems of the flight controller on the base of proposed integration technology.

\section{DESCRIPTION OF MAJOR NAVIGATION SYSTEMS AND THEIR CHARACTERISTICS}

Due to the fact that the UAV is limited in size and the maximum payload currently uses MEMS technology which provides low cost and navigation problem solution, but with limited accuracy.

The main modules of navigation systems are:

a) Module of intertial navigation system (INS) MPU6050 is used to determine the location of the UAV in space, its angular velocities and accelerations. It is constructed on the basis of at least one 3-axis gyro and 3-axis accelerometer. The disadvantage of this system is the non linear continuous accumulation of static error. Minimal requirements for accuracy - gyroscopic MEMS sensor STMicroL3GD20H 16 bit with the sensitivity of the gyro $16 \mathrm{~g}$ and STMicroLSM303D 14-bit accelerometer / magnetometer 2 in 1 Crystal $16 \mathrm{~g}$.

The minimal required precision of gyroscopes and accelerometers as the INS module is shown below:

- operating voltage $2.375 \mathrm{~V} \ldots 3.4 \mathrm{~V}$;

- the accuracy measurement of gyro $\pm 250 \pm 500 \pm 1000$ $\pm 2000 \%$;

- the sensitivity of the gyro131 65.5 32.8 16.4 LSB \%

- the accuracy of the accelerometer $\pm 2 \pm 4 \pm 8 \pm 16 \mathrm{~g}$;

- the sensitivity of the accelerometer16834 81924096 2048;

- digital output $\mathrm{I}^{2} \mathrm{C}$ or SPI;

- logic voltage1.71 to VDD V;

- module dimensions $4 \times 4 \times 0.9 \mathrm{~mm}$.

b) Module of satelite navigation system (SNS) uBloxGPSNEO is used to determine UAV in space, its airspeed, coordinates and bind to them. SNS is based on GPS modules. The main disadavantag is very slight interferenceresistance and weak reliability. Therefore, it is often used as an error correction for INS. below:

The minimal required parameters of SNS module are listed

- ublox LEA-6H module;

- data frequency $5 \mathrm{~Hz}$;

- $\quad$ size $38 \times 38 \times 9 \mathrm{~mm}$;

- filters LNA and SAW;

- rechargeable LIPO battery;
- low noise $3.3 \mathrm{~V}$ regulator;

- I2C EEPROM for configuration storage

- LED indicators for power and capture satellites;

- protective cover;

- compatible with APM 6-pin DF13 jack;

- weight $16.8 \mathrm{~g}$.

c) Module of aeromagnetometric system LSM303D+ airspeed sensor $3 D R 4525 D O$. It consists of a magnetometer (compass) and airspeed sensor - Pitot tube. It is used to determine the direction of flight (heading) as well as an emergency return to the take-off point, if necessary, and also provides data about the flight speed. The disadvantage of this system is the low accuracy of the magnetometer, error of $10 \ldots 12$ degrees, which is at distance of over $10 \mathrm{~km}$ from the start point of the UAV generates a very large sector that is uncomfortable under its search, due to the magnetic anomalies it is often simply fails temporarily and gives false readings. The minimal characteristics of precision corresponds LSM303D.

The minimal required precision of magnetometer as module is shown below.

Three-axis magnetic field sensor and acceleration:

- the magnetic field of $\pm 2 / \pm 4 / \pm 8 / \pm 12$ gauss;

- built-in 16-bit ADC;

- interface SPI / I2C;

- power supply from 2.16 to $3.6 \mathrm{~V}$;

- integrated temperature sensor;

- operating temperature $-40 \ldots 85^{\circ} \mathrm{C}$;

- built-in memory of a FIFO;

- housing LGA-16 $(3 \times 3 \times 1 \mathrm{~mm})$.

Airspeed sensor of aeromagnetometric module $4525 \mathrm{DO}$

With an integrated sensor with a measuring range 4525DO 1 psi (about $100 \mathrm{~m} / \mathrm{s}$ or $360 \mathrm{~km} / \mathrm{h}$ ) Pixhawk Airspeed Sensor Kit has a resolution of $0.84 \mathrm{~Pa}$, the resulting data have a resolution of 14 bit raw data come from the delta-sigma ADC with 24-bit resolution. As the sensor measures temperature to calculate the true airspeed of the air speed using sensor MS5611 static pressure Pixhawk. This sensor isn't affected by the heat of the surrounding components, so it more accurately shows the air temperature than previously produced analog sensors. It supports all versions of cards Pixhawk and PX4. Mounting holes M3 / 6-32.

Equipment includes:

- airspeed sensor;

- rubber tube;

- Pito tube;

- 4-cored cable $\mathrm{I} 2 \mathrm{C}$ bus. 
d) Terrain-Referenced Navigation Module. It is realized on basic of barometricsystem MEASMS5611 provides evaluation data of altitude, and laser rangefinders particularly effective during takeoff and landing where plantings $2 \ldots 3 \mathrm{~m}$ error of conventional MEMS barometers are critical. The high precision altimeters whose operating range reaches $1000 \ldots 1500 \mathrm{~m}$ perfectly serve to solve the problem of flight relief terrain, but are expensive and used only with the acute need for high precision. The minimal required accuracy barometric system is shown below:

- $\quad$ high resolution pressure 24 bit $\approx 0,0024$ mbar;

- $\quad$ resolution temperature $<0,01^{\circ} \mathrm{C}$;

- high resolution pressure $0.012 \mathrm{mbar}$

- altitude resolution $10 \mathrm{~cm}$;

- pressure range 10 to $1200 \mathrm{mbar}$;

- temperature range -40 to $85^{\circ} \mathrm{C}$;

- $\quad$ supply voltage 1.8 to $3.6 \mathrm{~V}$;

- $\quad$ low power (stand by: $\max .0 .14 \mu \mathrm{A}$ );

- $\quad$ excellent long term stability;

- $\quad \mathrm{I}^{2} \mathrm{C}$ - and SPI-Interface;
- High precision through individually compensated coefficients;

- $\quad$ ESD protected, HBM $4 \mathrm{kV}$;

- QFN package: $(5.0 \times 3.0 \times 1.0 \mathrm{~mm} 3)$;

- $\quad$ RoHS-compatible and $\mathrm{Pb}$ free.

e) Landscape Navigation System Module - optical system PX4 OPTICAL FLOW and as the system flight across the landscape in the form of a stereo pair of cameras with rectification mapping and building depth map that make the images from the cameras are then stitched into a large map that serves as an additional reference point to navigate the UAV.

\section{V.REVIEV OF MODERn FLIGHT AND NAVIGATION COMPLEXS}

Modern FNC module that can meet the above functionality requirements must have the following modular navigation systems: INS, SNS, AMNS, SARNS, TRNS and LNS.

barometric system aeromagnetometric system as the main sources of navigation information, and optical camera and camera systems terrain following for the flight as an extra.

The series-manufactured FNC with sets of available navigation systems is represented in Table I.

TABle I Series-Manufactured FNC With Sets of AVAIlable NAVIGATION Systems

\begin{tabular}{|c|c|c|c|c|c|c|c|c|c|c|}
\hline \multicolumn{11}{|c|}{ Autopilot Feature Matrix } \\
\hline Features & Lisa 1 v1.1 & $\begin{array}{l}\text { Lisa } / M \\
\text { v2.0 }\end{array}$ & Lisa/S & LinAM V4 & KroozSD & $\begin{array}{l}\text { Apogee } \\
\text { v1.00 }\end{array}$ & $\begin{array}{l}\mathrm{Um} \\
\mathrm{v} 1.0\end{array}$ & & Pixhawk & NavStick \\
\hline \multicolumn{11}{|c|}{$\mathrm{MCU}$} \\
\hline Part & $\begin{array}{l}\text { STM32F1 } \\
\text { 03RE }\end{array}$ & $\begin{array}{l}\text { STM32F105 } \\
\text { RCT6 }\end{array}$ & $\begin{array}{l}\text { STM32F103 } \\
\text { REY6 }\end{array}$ & $\begin{array}{l}\text { STM32F405 } \\
\text { RGT6 }\end{array}$ & $\begin{array}{l}\text { STM32F405R } \\
\text { GT6 }\end{array}$ & $\begin{array}{l}\text { STM32F40 } \\
\text { GT6 }\end{array}$ & & $\begin{array}{l}\text { LPC214 } \\
8\end{array}$ & $\begin{array}{l}\text { STM32 } \\
\text { F427 }\end{array}$ & $\begin{array}{l}\text { STM32F41 } \\
\text { 5RG }\end{array}$ \\
\hline Clock & $72 \mathrm{MHz}$ & $72 \mathrm{MHz}$ & $72 \mathrm{MHz}$ & $168 \mathrm{MHz}$ & $168 \mathrm{MHz}$ & $168 \mathrm{MHz}$ & & $60 \mathrm{MHz}$ & $\begin{array}{l}168 \mathrm{MH} \\
\mathrm{z}\end{array}$ & $168 \mathrm{MHz}$ \\
\hline Flash & $512 \mathrm{~KB}$ & $256 \mathrm{~KB}$ & $512 \mathrm{~KB}$ & $1024 \mathrm{~KB}$ & $1024 \mathrm{~KB}$ & $1024 \mathrm{~KB}$ & & $512 \mathrm{~B}$ & $1024 \mathrm{~KB}$ & $1024 \mathrm{~KB}$ \\
\hline RAM & $64 \mathrm{~KB}$ & $64 \mathrm{~KB}$ & $64 \mathrm{~KB}$ & $192 \mathrm{~KB}$ & $128 \& 64 \mathrm{~KB}$ & $128 \& 64 \mathrm{KH}$ & & $\begin{array}{l}32 \mathrm{~KB} \& \\
8 \mathrm{~KB}\end{array}$ & $192 \mathrm{~KB}$ & $192 \mathrm{~KB}$ \\
\hline \multicolumn{11}{|c|}{ Onboard Sensors } \\
\hline $\begin{array}{l}\text { MEMS } \\
\text { IMU }\end{array}$ & no & aspirin & yes & yes & Kroozext & yes & & yes & yes & yes \\
\hline $\begin{array}{l}\text { Magnetome } \\
\text { ter }\end{array}$ & no & no & yes & yes & yes & yes & & no & yes & yes \\
\hline Barometer & yes & yes & yes & yes & yes & yes & & yes & yes & yes \\
\hline $\begin{array}{l}\text { Diff } \\
\text { Pressure }\end{array}$ & yes & no & no & yes & no & no & & no & yes & optional \\
\hline GPS & no & no & yes & yes & no & no & & no & yes & optional \\
\hline \multicolumn{11}{|c|}{ Input Output Communication Interfaces } \\
\hline UART & $3 \& 1 \mathrm{RX}$ & $2 \& 2 \mathrm{RX}$ & $1 \& 1 \mathrm{RX}$ & $1 \& 2 \mathrm{RX}$ & 3 & $3 \& 1 \mathrm{RX}$ & & 2 & 5 & $4^{9}$ \\
\hline I2C & 2 & $1+1^{5}$ & $1^{8}$ & 1 & 2 & 2 & & 2 & 8 & $2^{9}$ \\
\hline SPI & 2 & 1 & 0 & 0 & 1 & 1 & & 1 & 4 & $1^{9}$ \\
\hline $\mathrm{ADC}$ & 3 (12bit) & $3+2(12 b i t)^{5}$ & 0 & 3 (12bit) & $4+1(12 b i t)^{5}$ & $0+3$ (12bit & & $0+4$ (10bit) & 3 (12bit) & $2^{9}$ \\
\hline PWWI & 6 & $6+2^{5}$ & 6 & 8 & $10+1^{5}$ & $6+1$ & & 6 & 16 & $6^{9}$ \\
\hline $\begin{array}{l}\text { PPM } \\
\text { Output }\end{array}$ & no & no & no & no & no & no & & no & yes & no \\
\hline $\begin{array}{l}\text { PPM } \\
\text { Capture }\end{array}$ & 1 & $0+1^{5}$ & 1 & $6^{9}$ & 1 & $1+1^{5}$ & & $1+1^{5}$ & $6^{9}$ & 1 \\
\hline
\end{tabular}




\section{Problem STATEMENT}

Development of navigation units is connected with great difficulties because it requires time-consuming and costly, so from our point of view requires a methodology based on the known elements of the world's major manufacturers: ST microelectronics, Gumstix, Analog Device, Beagle Bone and others. In this case, it is required to be able to develop a methodology for building the $\mathrm{FNC}$, that provides aggregation selected navigation systems for the solution of predetermined problems.

Let we have $m$ navigation systems that provide the estimation of navigation parameters and functioning on different physical principles. It is required to choose optimal $n$ navigation systems $(n \leq m)$, which in turn should have a technical compatibility, and choose the type of their integration in integration navigation complex basic model from which will be produced aggregation.

\section{CHOiCE JUSTIFICATION OF INTEGRATED FNC}

Structure Based on Flight Controller PiXHAWK

After analyzing the necessary functionality of FNC in its composition must be include the following systems: INS, SNS, AMNS as the main source of navigational information, as well as SARNS, TRNS and LNS as additional.

From the table comparisons of different FNC you can see that not all of them contain all above navigation systems, furthermore some FNS don't have the ability of external navigation systems connection as sources of additional information and the correction of the flight.

Beside that is that most companies - manufactures of FNC do not provide its open source code and access to the hardware.
Therefore, as a solution of given problem is offered to choose a flight controller Pixhawk as a main computing unit of FNC. It consists of the INS, AMNS and TRNS. Satellite navigation system is connected by separate external module, which has a second outer compass for averaging errors and improve the accuracy of readings, as well as a complete technical compatibility. But the most important thing this FNC has a large number of open external interfaces, such as I2C, SPI, UART, CAN, for connecting additional external modules of navigation systems. The program architecture of FNC Pixhawk (Fig. 1) is organized as state machine, with states its flight and its logic flight control scheme is as follows.

This FNC working on the real time operating system NuttXOS and has a second co-processor for monitoring the operability of all navigation modules every second. In case obtaining the messages from co-processor about inoperability of SNS or it's failure for several seconds, the state machine makes a decision: to continue the mission, or waiting until the signal resumes, or return to the point of takeoff. In addition Pixhawk uses the main processor STM32F427 CortexM4 clock speed of $168 \mathrm{Mhz}$, and maximum computational load currently only near $10 \%$. This allows extension of the FNC by additional navigation systems and data processing.

To increase navigational accuracy of FNC Pixhawk it should be adding by SARNS, TRNS and LNS connecting a set of cameras to frame of image processing and connecting the optical camera with a frequency of at least 240 shots of frames per second to duplicate relation to the coordinates in the case of temporary failure SNS.

The main advantage of this FNC is the possibility of programming its actions on the events of failure for each of the navigation modules separately, and a second co-processor continuously monitors in mile per seconds the state of all modules in the integrated navigation FNC.

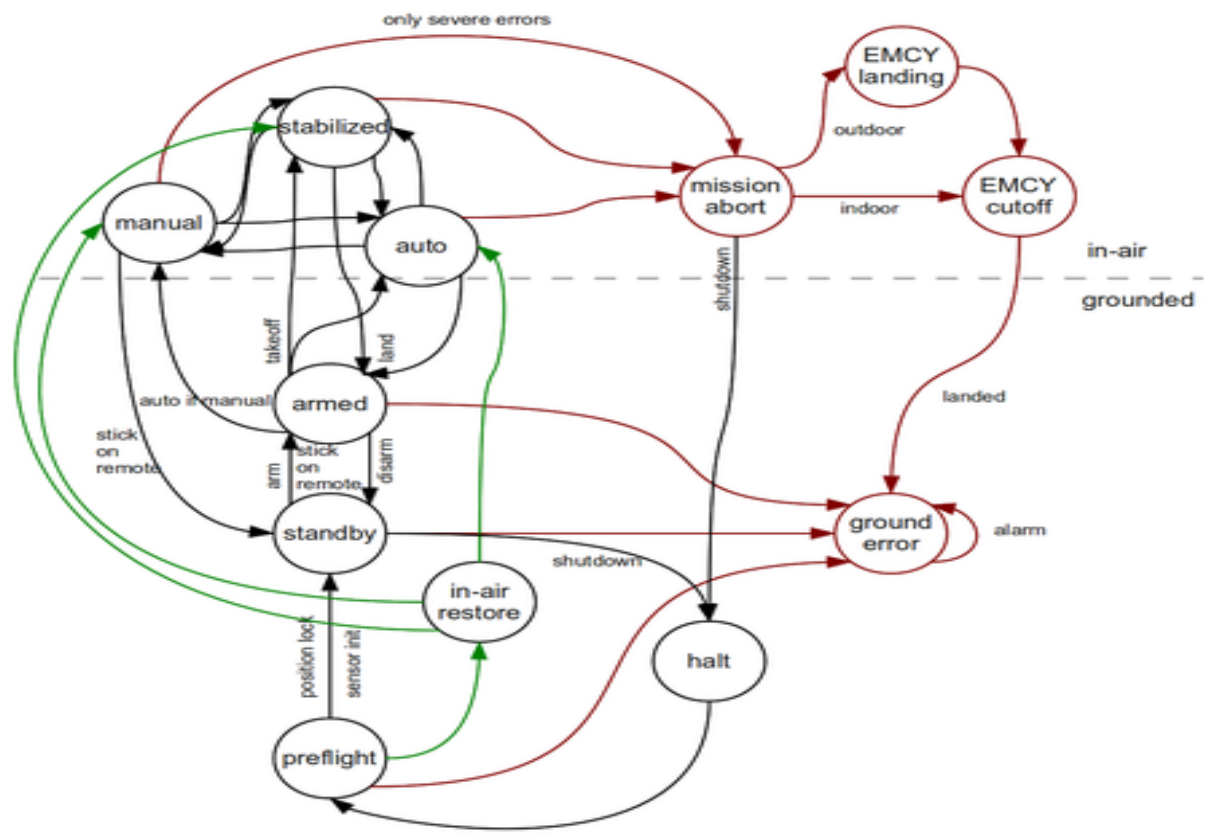

Fig. 1. Program architecture of logic FNC Pixhawk. 


\section{Features of the flight controller Pixhawk:}

Enhanced 32-bit ARM Cortex ${ }^{\circledR}$ - M4 clocked Nutt X RTOS 14 PWM / servo outputs ( 8 with failsafe and manual control, 6 auxiliary, high-).

A large number of connection options for additional peripherals (UART, I2C, CAN)):

- a huge margin of $90 \%$ of the computing power available;

- a large number of internal and external interfaces;

- Build on the basis of the flight controller, a big plus is that the flight controller as open source, and every second of the flight and during the execution of commands, we know that to expect from him;

- Constant monitoring of fault tolerance to the second processor and can be programmed actions whenever possible failures;

- Real-time operating system with a good GUI interface and self-powering;

External safety button for easy switching engines

High performance, multi-tone Piezo audio indicator microSD card for long recording Flight logs (black box).

\section{PIXHAWK SPECIFICATIONS}

- Processor

- 32-bit ARM Cortex M4 core with FPU

- $168 \mathrm{Mhz} / 256 \mathrm{~KB}$ RAM/2 MB Flash

- 32-bit failsafe co-processor

- Sensors

- MPU6000 as main accel and gyro

- ST Micro 16-bit gyroscope

- ST Micro 14-bit accelerometer/compass (magnetometer)

- MEAS barometer

- Power

- Ideal diode controller with automatic failover

- Servo rail high-power (7 V) and high-current ready

- All peripheral outputs over-current protected, all inputs ESC protected

\section{- Interfaces}

- 5x UART serial ports, 1 high-power capable, $2 \mathrm{x}$ with HW flow control

- Spektrum DSM/DSM2/DSM-X Satellite input

- Futaba S.BUS input (output not yet implemented)

- PPM sum signal

- RSSI (PWM or voltage) input

- I2C, SPI, 2x CAN, USB
- $\quad 3.3$ and 6.6 ADC inputs

\section{- Dimensions}

- Weight $38 \mathrm{~g}(1.3 \mathrm{oz})$

- Width $50 \mathrm{~mm}(2.0$ ”)

- Height $15.5 \mathrm{~mm}\left(.6^{\prime \prime}\right)$

- Length $81.5 \mathrm{~mm}(3.2 ”)$

VIII. TeChNOLOGy InTEgRATION NAVIGATION MODUleS TO THE CONTROLLER

This FNC has the following set of external interfaces (Fig. 2).

Navigation modules are integrated with the FNC by the interfaces above, but more often they are I2S or SPI serial interface, that's why there are bus extenders as I2C extender and SPI extender for connection any number of modules to FNC. For technically comfortable connection external navigation modules to FNC are used DF13 4 excretory and 6 connectors.

\section{INTEGRATION ARCHITECTURE}

There are many ways of combining information from multiple navigation systems [3]. The design of the integration architecture is a tradeoff between maximizing the accuracy and robustness of the navigation solution, minimizing the complexity, and optimizing the processing efficiency. It must also account for the characteristics of the different navigation technologies.

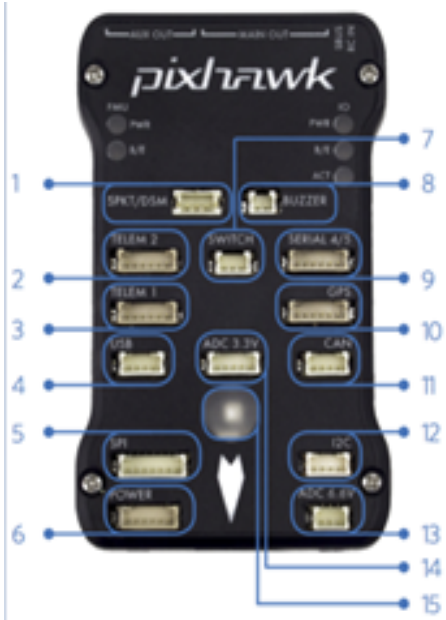

1 Spectrum DSM receiver 2 Telemetrv(on screen disolav)

3 Telemetry(radio telemetry) 4 USB

5 SPI bus interface

6 Power module

7 Safety switch motor button 8 Buzzer

9 Serial

10 GPS module IO

11 CAN bus interface

$12 \mathrm{I} 2 \mathrm{C}$ splitter module interface

13 Analog to digital converter $6.6 \mathrm{~V}$

14 Analog to digital converter $3.3 \mathrm{~V}$

15 Led indicator

Fig. 2 External interfaces of FNC.

Schemes of connection modules to FNC (Figs 3 - 5).

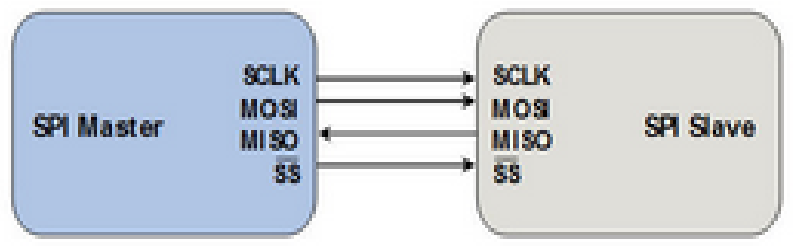

Fig. 3. Serial interface. 


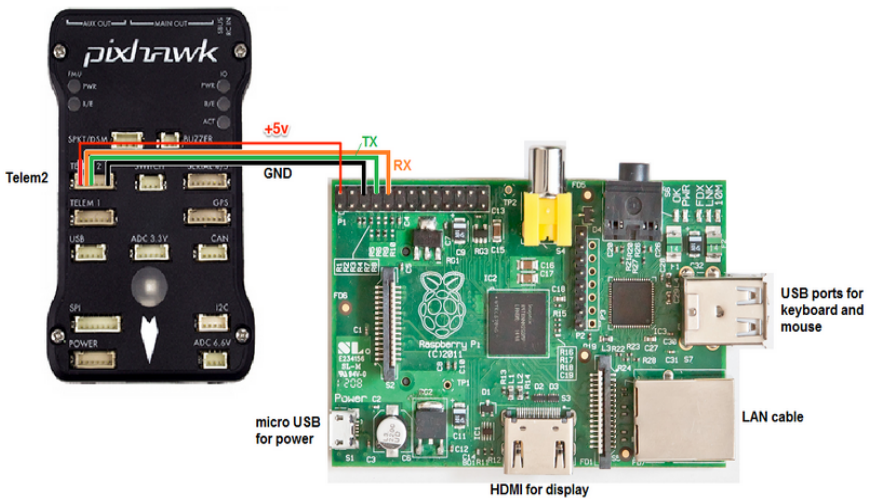

Fig. 4. USART interface.

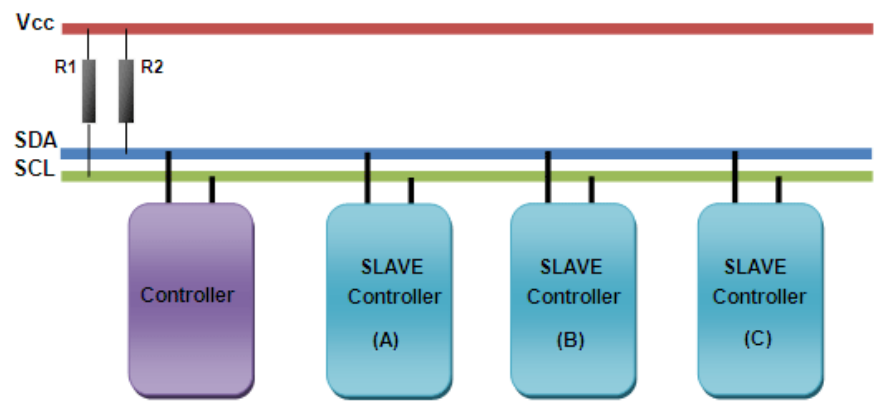

Fig. 5. Multi serial interface.

In a federated-filters integration architecture, a reference inertial or dead-reckoning navigation solution is integrated separately, with each of the aiding navigation systems in a bank of local Kalman filters. Each local filter's integration with its navigation sensors may be centralized or cascaded.
There are a number of different ways in which the local filter outputs may be combined to produce an integrated navigation solution. The no-reset (FNR), fusion-reset (FFR), zero-reset (FZR), and cascaded versions of federated integration are described next.

Different architectures may be used for different sensors in the same integrated navigation system, provided the final stage of the processing chain is common. Thus, the least-squares, FNR, and FFR architectures can be mixed, as can the centralized, cascaded, FZR, and federated-cascaded

architectures. However, architectures using a snapshot least-squares fusing algorithm to produce the integrated navigation solution cannot be mixed with architectures using a Kalman filter. Hybrid architectures are typically used where constraints in the design of the constituent navigation systems prevent use of the desired architecture in all cases.

\section{CONCLUSION}

There are substantiated the necessity of creating integrated navigation complex for UAV basis on of flight controller Pixhawk. Was shown that this FNC allows building the navigation complex with high accuracy and noise immunity due to possibility of connection additional navigation modules to it.

\section{REFERENCES}

[1] V.A. Rogoshyn, V.M. Sineglazov and N.K. Filyashkin, Flight Navigation Complexes of Aircrafts. 2004, Kyiv, NAU, 238 p. (in Ukrainian)

[2] Flight controller pixhawk http://pixhawk.org

[3] Paul D. Groves, Principles of GNSS, Inertial, and Multisensor Integrated Navigation Systems. 2008, 503 p. 